\title{
A model of knowledge mapping in visualizing the hotspot of dengue
}

\begin{abstract}
Formulation process is very important in model development. Due to that, this study was performed as a formulation process for the K-Map model development in order to visualize the dengue hotspot. Purpose of this study is to find the appropriate technique on knowledge visualization and also to identify the contributing parameters for the dengue outbreak. Methodology that has been used is by analysing the literature review from empirical paper. Result from this study shows that Venn diagram is one of the technique that can be used to visualize the dengue hotspot while the environmental parameter is one of the contributing factors in dengue transmission and distribution. Ten environmental parameters that are land use, housing types, Land Surface Temperature, Elevation, Soil Moisture, Greenness, Population Density, rainfall, temperature and humidity have been identified. As a conclusion, climate change impact is one of the environmental parameter, which influences the trend of dengue transmission and distribution. The impact of climate change on the transmission patterns of dengue diseases can be both direct and indirect. A direct impact describes how the changes in precipitation and temperature trend can influence the trend of the dengue transmission and distribution pattern. Instead indirect impact describe how the factors such as human population dynamics and their effects on exposure risk, changes in vegetation, hydrology and other landscape features can influence the trend of dengue transmission and distribution pattern.
\end{abstract}

Keyword: Literature review; Environmental parameter; Knowledge map; Knowledge visualization; Dengue hotspot 